\title{
Epidemiology and clinical characteristics of 43 COVID-19 patients in Weifang, China
}

\author{
Qingxiu Wang ${ }^{1}$, Shiliang Zheng ${ }^{2}$, Wei Tan ${ }^{3}$, Li Qi $^{4}$, Weiguang Shao ${ }^{5}$, Ming Zhang ${ }^{6}$, Xiaodong Sun ${ }^{7}$ \\ ${ }^{1}$ Department of Infection Management, ${ }^{2}$ Department of General Practice, Affiliated Hospital of Weifang Medical University, Weifang, China; \\ ${ }^{3}$ Department of Respiration Medicine, Weifang People's Hospital, Weifang, China; ${ }^{4}$ Department of Infectious Disease, ${ }^{5}$ Department of Medical \\ Imaging, ${ }^{6}$ Department of Critical Care Medicine, ${ }^{7}$ Department of Endocrinology, Clinical Research Center, Affiliated Hospital of Weifang Medical \\ University, Weifang, China \\ Contributions: (I) Conception and design: Q Wang, S Zheng; (II) Administrative support: Q Wang, S Zheng; (III) Provision of study materials or \\ patients: Q Wang, S Zheng, W Tan, L Qi, M Zhang; (IV) Collection and assembly of data: Q Wang, S Zheng, W Tan, L Qi, W Shao, M Zhang; (V) \\ Data analysis and interpretation: S Zheng; (VI) Manuscript writing: All authors; (VII) Final approval of manuscript: All authors. \\ Correspondence to: Shiliang Zheng, MS. Department of General Practice, Affiliated Hospital of Weifang Medical University, No. 2428 Yuhe road, \\ Kuiwen District, Weifang 261031, China. Email: wyfyqkyx@163.com.
}

Background: To investigate the epidemiology and clinical characteristics of patients infected with coronavirus disease 2019 (COVID-19) in Weifang, China.

Methods: The demographic data of 43 COVID-19 patients identified in Weifang were used to investigate whether they had traveled to epidemic areas and whether they had close contact with confirmed cases. On admission, patients' symptoms and results of laboratory tests and imaging were analyzed.

Results: Among the 43 COVID-19 patients. including 9 third generation infected cases, 16 (37.2\%) were imported, who infected the rest. Most cases were middle-aged with approximate sex ratio. A "super spreader", Mr. Zhang made it necessary to quarantine 69 medical personnel. Mr. Zhang directly infected six individuals who, in turn, infected another six individuals. Another patient, Mr. Wang, spread the infection to his five family members at a family gathering. Subsequently, the daughter infected her husband. The most common COVID-19 symptoms were fever, weakness, dry cough, and cough sputum. In most patients, white blood cell counts were not elevated and lymphocyte counts were decreased. Elevated C-reactive protein and serum amyloid A protein (SAA) levels were commonly observed. There was no death among the patients or infection among the medical staff.

Conclusions: The infection by the COVID-19 in Weifang was mostly the result of close contact with imported cases. These circumstances underscore the need to comprehensively strengthen the management for patients to prevent and control the spread of the virus.

Keywords: Coronavirus disease 2019 (COVID-19); epidemiology; clinical characteristics; third-generation infection; super spreader

Submitted Mar 03, 2020. Accepted for publication Jul 09, 2020.

doi: 10.21037/apm-20-537

View this article at: http://dx.doi.org/10.21037/apm-20-537

\section{Introduction}

In December 2019, there was an outbreak of pneumonia associated with fever and expectoration, and human to human transmission was found $(1,2)$. In most cases, symptoms improved quickly, however, some patients developed severe acute respiratory syndrome, often leading to acute respiratory failure. On January 7, 2020, a novel coronavirus (2019-nCoV) was identified by the Chinese Center for Disease Control and Prevention from patient throat swab samples. The illness caused by this virus was subsequently named coronavirus disease 2019 (COVID-19) by the World Health Organization (3). 
With the Chinese New Year and the Spring Festival approaching, many non-residents left Wuhan for home, resulting in the rapid spread of COVID-19 in China. On January 23, 2020, the first case was identified in Weifang, Shandong Province. By February 16, 2020, a total of 43 cases had been diagnosed, and all patients were hospitalized. At the time of writing, nine patients had recovered following treatment and been discharged. The goal of the present study was to analyze the epidemiology and clinical characteristics of 43 patients with COVID-19 diagnosed in Weifang. We present the following article in accordance with the STROBE checklist (available at http://dx.doi. org/10.21037/apm-20-537).

\section{Methods}

\section{Study design and subjects}

A total of 43 patients with COVID-19 were hospitalized in Weifang from January 23, 2020, to February 16, 2020, and were enrolled. This study was approved by institutional ethics board of Affiliated Hospital of Weifang Medical University (No. 2020003). Written informed consent was obtained from all patients for publication of this study. The study conformed to the provisions of the Declaration of Helsinki (as revised in 2013).

\section{Procedure}

Epidemiological and demographic data were obtained from the daily notices of the Weifang Health Commission. Clinical characteristics, laboratory results, treatments, and prognostic data were obtained from the patients' medical records. Clinical results were followed up to February 16, 2020. Information not included in the records was obtained by direct communications with the attending physician and other health care providers.

Throat swab samples from the upper respiratory tracts of all patients were stored in viral collection medium, and the presence of 2019-nCoV was determined by real-time RT-PCR in the Weifang Center for Disease Control and Prevention Laboratory. Sputum samples were collected on admission and cultured to identify pathogenic bacteria or fungi, and routine drug sensitivity tests were conducted for bacteria. Additionally, all patients underwent a chest computed tomography (CT) examination, the blood routine, $\mathrm{C}$-reactive protein and serum amyloid a protein (SAA), which are all indicators of infection, were examined.

\section{Clinical results}

Patients were classified as COVID-19 based on their arrival from Wuhan or other epidemic areas, close contact with individuals from the epidemic areas or confirmed personnel, and diagnostic data. A synopsis of the symptoms, complications, laboratory results, respiratory pathogens, and chest CT results were prepared. The diagnosis and treatment followed the rapid advice guideline for the diagnosis and treatment of 2019-nCoV associated pneumonia (4). The treatment plan was adjusted based on changes in patient conditions and was supplemented with Chinese medicines (Reduning or Xiyanping). Patient prognoses and the infection status of medical staff were continuously monitored.

\section{Statistical analysis}

Continuous data were presented as the mean and standard deviation. Non-normally distributed data were presented as the median and interquartile range, and count data were presented as $\mathrm{n}(\%)$. All analyses were performed using SPSS (version 26.0, Chicago, IL, USA).

\section{Results}

\section{Patient demographic information}

The ages of the 43 patients infected with COVID-19 ranged from 16 months to 70 years. Most patients were middleaged, with 31 to 40 years old constituting the largest group and 41 to 50 years old the second largest group. There were comparable numbers of male and female patients. The patients included farmers, staff, self-employed individuals, retirees, students, teachers, drivers, worker and unemployed individuals. A fraction of the patients had underlying diseases, including hypertension, diabetes, chronic obstructive pulmonary disease, ischemic heart disease, chronic hepatitis, and Parkinson's syndrome (Table 1).

\section{Epidemiologic characteristics}

Among the 43 COVID-19 patients, 16 (37.2\%) represented imported cases. From January 23, 2020 to February 3, 2020, 14 newly confirmed cases in Weifang were all imported: 11 represented arrivals from Wuhan, and three represented arrivals from Bengbu, Anhui Province. One patient returned to Weifang from Huanggang, Hubei Province on February 3, 2020, and was hospitalized and diagnosed on February 5. 
Table 1 Patients characteristics

\begin{tabular}{|c|c|}
\hline Basic information & Values \\
\hline \multicolumn{2}{|l|}{ Age (years), n (\%) } \\
\hline Average (range) & $42.82 \pm 11.24(1.3-70.0)$ \\
\hline$\leq 20$ & $1(2.3)$ \\
\hline $21-30$ & $5(11.6)$ \\
\hline $31-40$ & $15(34.9)$ \\
\hline $41-50$ & $10(23.3)$ \\
\hline $51-60$ & 7 (16.3) \\
\hline $61-70$ & $5(11.6)$ \\
\hline$\geq 71$ & $0(0.0)$ \\
\hline \multicolumn{2}{|l|}{ Gender, n (\%) } \\
\hline Male & $23(53.5)$ \\
\hline Female & $20(46.5)$ \\
\hline \multicolumn{2}{|l|}{ Occupation, n (\%) } \\
\hline Farmer & $9(20.9)$ \\
\hline Staff & $12(27.9)$ \\
\hline Self-employed individuals & $10(23.3)$ \\
\hline Retiree & $3(7.0)$ \\
\hline Student & $3(7.0)$ \\
\hline Teacher & $1(2.3)$ \\
\hline Driver & $1(2.3)$ \\
\hline Unemployed individual & $2(4.7)$ \\
\hline Others & $2(4.7)$ \\
\hline \multicolumn{2}{|l|}{ Chronic disease, n (\%) } \\
\hline Diabetes & $5(11.6)$ \\
\hline Hypertension & $9(20.9)$ \\
\hline Chronic obstructive pulmonary disease & $2(4.7)$ \\
\hline Parkinson's syndrome & $1(2.3)$ \\
\hline Ischemic heart disease & $2(4.7)$ \\
\hline Chronic hepatitis & $2(4.7)$ \\
\hline Ischemic cerebrovascular disease & $3(7.0)$ \\
\hline
\end{tabular}

One imported case returned to Weifang from Yantai.

The remaining 27 patients $(62.8 \%)$ were infected by imported cases. Mr. Zhang, who was identified by the Chinese media and dubbed the "super spreader" traveled to Bengbu, Anhui Province, on January 17, 2020, and had close contact with individuals in Wuhan on January 18.
After returning to Weifang on the evening of January 20, he developed fever and headache and sought medical treatment at a hospital the next day. He deliberately concealed his personal history of travel and contacts with infected individuals and was admitted to the hospital with a diagnosis of pneumonia. Between January 28 and January 30, he visited relatives in the intensive care unit of the hospital. His diagnosis with COVID-19 on February 2 made it necessary to put 69 medical personnel in quarantine. Without wearing a mask, he appeared in two populated areas and the $4 \mathrm{~S}$ car shop, making contact with 49 non-medical personnel, all of whom were subsequently quarantined. During this period, Mr. Zhang infected six additional individuals. One of them subsequently, infected two family members and another infected four family members. In total, there were 12 cases directly or indirectly infected by Mr. Zhang. After 14 days of medical observation, chest CT, and DNA analysis of throat swab samples, no infections were diagnosed among the 69 quarantined medical workers.

Moreover, one couple that socialized with Mr. Zhang was diagnosed with COVID-19. The husband also infected his aunt and cousin. Another patient who traveled to Weifang for a family gathering transmitted the virus to his wife, son, daughter-in-law, and two daughters, and one of the daughters then infected her husband. One of the other imported cases had close contact with a brother and infected two relatives, and another case was a couple who came in close contact with the virus. Two cases had no history of exposure and the source of infection was unknown.

In summary, 27 individuals were infected by 16 imported cases in Weifang, an indication that one infected person can infect, on average, 1.69 individuals. Moreover, patients infected by imported cases can infect others (i.e., third generation transmission). At present, nine third-generation infected patients $(20.9 \%)$ have been identified, including one patient with severe illness.

\section{Clinical characteristics}

\section{Clinical symptoms}

The major symptoms of the 43 patients were fever, weakness, dry cough, sputum production, muscle pain, and headache. Some patients experienced shortness of breath, sore throat, stuffy nose, runny nose, diarrhea, nausea and vomiting, tachypnea, confusion, and chest pain. In two cases, fever and cough were not obvious and COVID-19 was diagnosed only during the period of medical observation (Table 2). 
Table 2 Clinical symptoms of 43 patients with COVID-19

\begin{tabular}{lc}
\hline Symptoms & Number (\%) \\
\hline Clinical symptoms & $38(88.4)$ \\
Fever & $29(67.4)$ \\
Dry cough & $23(53.5)$ \\
Sputum & $6(14.0)$ \\
Shortness of breath & $15(34.9)$ \\
Muscle pain & $39(90.7)$ \\
Weakness & $10(23.3)$ \\
Headache & $3(7.0)$ \\
Sore throat & $3(7.0)$ \\
Runny nose & $2(4.7)$ \\
Chest pain & $3(7.0)$ \\
Diarrhea & $2(4.7)$ \\
Nausea and vomiting & $41(95.3)$ \\
Two or more symptoms & $2(4.7)$ \\
Combined disease & \\
ARDS & $1(2.3)$ \\
Acute respiratory failure & \\
\hline
\end{tabular}

\section{Laboratory tests and imaging results}

Laboratory examinations were carried out in all 43 patients. At admission, the white blood cell counts of most patients were normal or slightly decreased or increased. In most cases, lymphocyte counts were below the normal range. C-reactive protein and SAA were commonly increased (Table 3). Chest CT revealed patchy shadows or groundglass opacities, or both, in the lungs of all patients; in most cases, CT changes progressed quickly and lesions were absorbed rapidly (Figure 1). Some patients initially showed unilateral lung changes that rapidly spread to both lungs. In a few, mild cases, changes were restricted to one lung.

\section{Therapy and prognosis}

All 43 patients were given ganciclovir or abidor as antiviral
Table 3 Laboratory and imaging results of 43 patients infected by COVID-19

\begin{tabular}{|c|c|}
\hline Blood routine examination & Number (\%) \\
\hline \multicolumn{2}{|l|}{ White blood cell count $\left[(3.5-9.5) \times 10^{9} / L\right]$} \\
\hline Increased & $4(9.3)$ \\
\hline Decreased & $6(14.0)$ \\
\hline \multicolumn{2}{|l|}{ Neutrophil count $\left[(1.8-6.3) \times 10^{9} / L\right]$} \\
\hline Increased & $4(9.3)$ \\
\hline Decreased & $3(7.0)$ \\
\hline \multicolumn{2}{|l|}{ Lymphocyte count $\left[(1.1-3.2) \times 10^{9} / L\right]$} \\
\hline Increased & $0(0.0)$ \\
\hline Decreased & $41(95.3)$ \\
\hline \multicolumn{2}{|l|}{ C-reactive protein $(0.0-5.0 \mathrm{mg} / \mathrm{L})$} \\
\hline Increased & 41(95.3) \\
\hline Decreased & $0(0.0)$ \\
\hline \multicolumn{2}{|l|}{ SAA } \\
\hline Increased & $40(93.0)$ \\
\hline Decreased & $0(0.0)$ \\
\hline 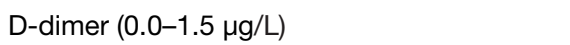 & $6(14)$ \\
\hline Increased & $10(23.3)$ \\
\hline Decreased & $0(0.0)$ \\
\hline \multicolumn{2}{|c|}{ Blood biochemistry alanine aminotransferase (9.0-50.0 U/L) } \\
\hline Increase & $10(23.3)$ \\
\hline Decrease & $2(4.7)$ \\
\hline \multicolumn{2}{|l|}{ Lactate dehydrogenase (120.0-250.0 U/L) } \\
\hline Increased & $12(27.9)$ \\
\hline Decreased & $0(0.0)$ \\
\hline \multicolumn{2}{|l|}{ Glucose (3.9-6.1 mmol/L) } \\
\hline Increased & $6(14.0)$ \\
\hline Decreased & $0(0.0)$ \\
\hline \multicolumn{2}{|l|}{ CT findings } \\
\hline Unilateral pneumonia & $8(18.6)$ \\
\hline Bilateral pneumonia & $35(81.4)$ \\
\hline Multiple mottling and ground-glass opacity & $25(58.1)$ \\
\hline
\end{tabular}



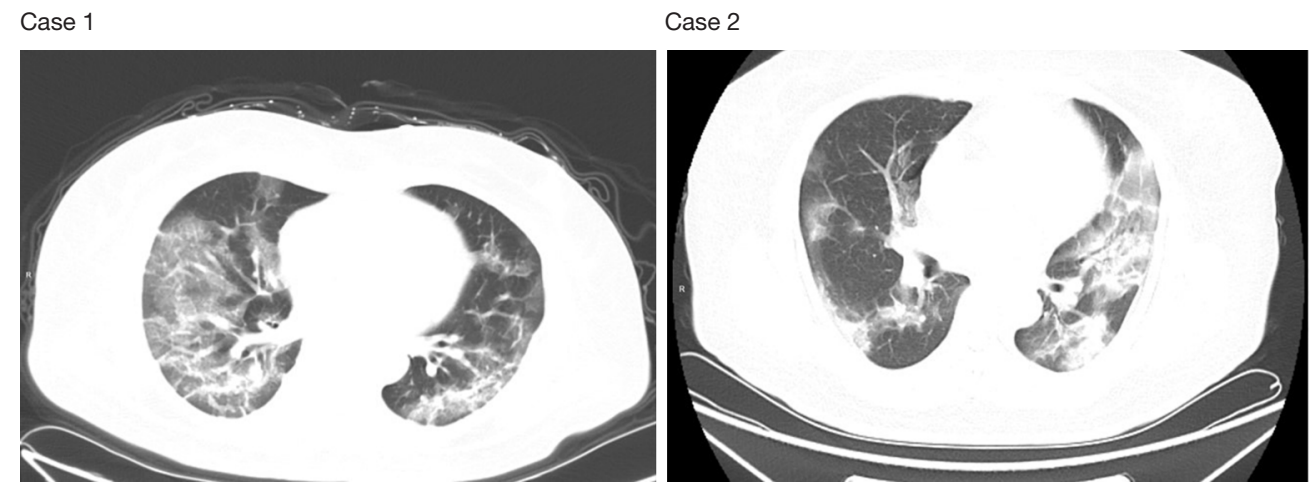

Figure 1 Chest CT image of two patients with COVID-19. Chest CT image revealed patchy shadows and ground-glass opacities.

therapy along with, injections of the traditional Chinese medicine Reduning or Xiyanping. Most patients were treated with antibiotics, methylprednisolone, interferon, and oxygen inhalation. Six patients received non-invasive mechanical ventilation for $2-10$ days and were given intravenous immunoglobulin $(10-15 \mathrm{~g})$ for $2-3$ days. Three patients with severe illness underwent endotracheal intubation and received mechanical ventilation 2-4 days later. One of these patients receirporeal membrane oxygenation (ECMO). Following treatment, one patient was stable and two remained in severe condition. As of February 16,2020 , nine of the 43 patients had been discharged, while the rest remained hospitalized.

\section{Discussion}

\section{The COVID-19 outbreak in Weifang}

The Spring Festival is the most important festival in China, and it is traditional to visit home during this time. Gathering in their hometowns facilitates the transmission of respiratory and other infections. With the Spring Festival approaching, many people returned to Weifang in mid to late January. At that time, the awareness of the infectious nature and implementation of protective measures against COVID-19 had already increased. Therefore, the first patient with COVID-19 was identified in Weifang on January 23, 2020, consistent with the return of imported cases infected in Wuhan during the Spring Festival, and similar to the situation in other cities in China.

\section{Epidemiology of COVID-19 in Weifang}

The COVID-19 patients in Weifang included comparable numbers of males $(n=20)$ and females $(n=23)$, consistent with published literature $(1,3,5)$. COVID-19 affects all age groups, indicating that people have limited or no immunity to the virus and they are generally susceptible regardless of their age. However, the initial infections in Weifang primarily affected middle-aged individuals, $34.9 \%$ of patients were $31-40$ years old, and most (58.1\%) were $31-50$ years old. This finding may be related to the high mobility of middle-aged individuals and, the wide range of their activities, resulting in higher risk of contact with infected individuals. In Weifang, the age distribution of COVID-19-infected patients was similar to that reported previously $(1,6)$.

Mr. Zhang drove to Bengbu, Anhui Province, where he had close contact with patients from Wuhan. When he returned, he developed a fever. It took 12 days, until February 2, 2020, for Mr. Zhang's disease to be diagnosed, indicating that the medical staff had insufficient understanding of the problem during the early stage of the COVID-19 epidemic. During this period, we had only begun to understand the infectivity of the $\mathrm{nCoV}-19$. Because Mr. Zhang has not been to Wuhan, and it was not stated in the third version of the New Coronavirus Pneumonia Diagnosis and Treatment Plan that regions outside Hubei also belonged to the epidemic area, his condition was not diagnosed in time. Moreover, when he developed symptoms, health care providers did not investigate the presence of $\mathrm{nCoV}-19$ in time, displaying a lack of vigilance. The above situation resulted in transmission of the virus to six individuals, one of who infected four others. The rate of third-generation infection is high and, if it continues, may lead to local outbreaks. The local Center for Disease Control and Prevention and the hospital took decisive steps to isolate 69 medical staff and 49 non-medical staff. 
Although the cost of this decision was high, the effect was satisfactory because no infections were identified among these personnel. These events also demonstrate that prevention and control of COVID-19 infections is a continuous process and, in some cases, treatments may not be adequate. Close attention must be paid to these patients.

Another patient, Mr. Wang, spread the infection to five family members through close contact during a family gathering. Subsequently, his daughter infected her husband. This spread was possible because family members were familiar with one another and believed everyone to be healthy, resulting in a lack of awareness of the risk of infection. The above phenomenon highlighted the necessity to strengthen public knowledge of COVID-19 and including the incubation period and transmissions of the virus. Family gatherings must be canceled. Finally, comprehensive knowledge of prevention and control of COVID-19 should be established. In addition, early detection of potentially infectious patients is imperative, especially those with sporadic contact histories. For some asymptomatic but potentially infectious patients, related clues and risk factors should be identified in time. Furthermore, big data, mobile communication tracing, and monitoring devices should be used to fully and timely grasp the activity of cases, and to sequester them in a timely manner. In cases of close contacts, strict medical observation should be implemented. Two patients were diagnosed with COVID-19 after several days of medical observation, demonstrating that rigorous medical observation management of close contacts was necessary.

\section{The clinical characteristics of COVID-19 infection in Weifang}

The main symptoms of COVID-19 in Weifang were fever, weakness, dry cough, sputum production, and muscle pain; these observations were consistent with published reports. Although many cases had a mild form of the disease, there were three cases $(7 \%)$ of severe illness, a rate consistent with other publications (7). In accordance with the New Coronavirus Pneumonia Diagnosis and Treatment Plan (trial version 5), the treatment protocol included a traditional Chinese medicine and optimal outcomes were obtained (8).

The three patients with severe illness received noninvasive mechanical ventilation, but their condition continued to deteriorate. Therefore, after 2-4 days, they were intubated and received mechanical ventilation. One of these patients was treated with ECMO. After active therapy, one patient was stable and two remained in severe condition. By February 16, 2020, 9 of the 43 patients had been discharged, while the rest remained hospitalized. Importantly, no medical personnel were infected. This is because the Weifang Municipal Government attaches great importance to the clinical treatment of COVID-19. Additionally, recognized experts in respiratory diseases, infectious diseases, and intensive care units were recruited from Weifang 3A hospitals to help with diagnosis and treatment. The use of telemedicine for consultations between critically ill patients and renowned experts in Shandong Province was implemented to ensure that these patients received a high standard of care. The hospital also recognizes the importance of assigning experienced doctors to the fever clinic, which actively diagnoses and treats febrile patients, identifies suspected cases, and reduces rates of infection for medical staff. In particular, the "super spreader", Mr. Zhang, was hospitalized in the respiratory ward, and medical staff were unaware of his COVID-19 positive status. Luckily, there were no cases of infection of medical personnel, indicating that wearing a mask and basic hand hygiene were effective protection and that the internal prevention and control measures in the hospital were satisfactory.

\section{Acknowledgments}

Funding: This work was supported by the Shandong Province TCM Science and Technology Development Plan Project (grant no.2011-222), the Weifang Science and Technology Development Project (grant no. 20132095) and the Weifang Science and Technology Development Project (grant no. 2011-68). We thank Liwen Bianji, Edanz Editing China (www.liwenbianji.cn/ac), for editing the English text of a draft of this manuscript.

\section{Footnote}

Reporting Checklist: The authors have completed the STROBE checklist (available at http://dx.doi.org/10.21037/ apm-20-537).

Data Sharing Statement: Available at http://dx.doi. org/10.21037/apm-20-537

Conflicts of Interest: All authors have completed the ICMJE uniform disclosure form (available at http://dx.doi. 
org/10.21037/apm-20-537). The authors have no conflicts of interest to declare.

Ethics Statement: The authors are accountable for all aspects of the work in ensuring that questions related to the accuracy or integrity of any part of the work are appropriately investigated and resolved. This study was approved by Institutional Ethics Board of Affiliated Hospital of Weifang Medical University (No. 2020003). Written informed consent was obtained from all patients for publication of this study. The study conformed to the provisions of the Declaration of Helsinki (as revised in 2013).

Open Access Statement: This is an Open Access article distributed in accordance with the Creative Commons Attribution-NonCommercial-NoDerivs 4.0 International License (CC BY-NC-ND 4.0), which permits the noncommercial replication and distribution of the article with the strict proviso that no changes or edits are made and the original work is properly cited (including links to both the formal publication through the relevant DOI and the license). See: https://creativecommons.org/licenses/by-nc-nd/4.0/.

\section{References}

1. Chen N, Zhou M, Dong X, et al. Epidemiological and clinical characteristics of 99 cases of 2019 novel coronavirus pneumonia in Wuhan, China: a descriptive

Cite this article as: Wang Q, Zheng S, Tan W, Qi L, Shao W, Zhang M, Sun X. Epidemiology and clinical characteristics of 43 COVID-19 patients in Weifang, China. Ann Palliat Med 2020;9(5):2993-2999. doi: 10.21037/apm-20-537 study. Lancet 2020;395:507-13.

2. Lu H, Stratton CW, Tang YW. Outbreak of pneumonia of unknown etiology in Wuhan, China: The mystery and the miracle. J Med Virol 2020;92:401-2.

3. Pediatric Branch of Hubei Medical Association; Pediatric Branch of Wuhan Medical Association; Pediatric Medical Quality Control Center of Hubei. Recommendation for the diagnosis and treatment of novel coronavirus infection in children in Hubei (Trial version 1). Zhongguo Dang Dai Er Ke Za Zhi 2020;22:96-9.

4. Jin YH, Cai L, Cheng ZS, et al. A rapid advice guideline for the diagnosis and treatment of 2019 novel coronavirus (2019-nCoV) infected pneumonia (standard version). Mil Med Res 2020;7:4.

5. Huang C, Wang Y, Li X, et al. Clinical features of patients infected with 2019 novel coronavirus in Wuhan, China. Lancet 2020;395:497-506.

6. Wang D, Hu B, Hu C, et al. Clinical Characteristics of 138 Hospitalized Patients With 2019 Novel CoronavirusInfected Pneumonia in Wuhan, China. JAMA 2020;323:1061-9.

7. Pan $\mathrm{Y}$, Guan $\mathrm{H}$, Zhou S, et al. Initial CT findings and temporal changes in patients with the novel coronavirus pneumonia (2019-nCoV): a study of 63 patients in Wuhan, China. Eur Radiol 2020;30:3306-9.

8. Yu F, Du L, Ojcius DM, et al. Measures for diagnosing and treating infections by a novel coronavirus responsible for a pneumonia outbreak originating in Wuhan, China. Microbes Infect 2020;22:74-9. 